\title{
Do colorectal cancer patients diagnosed as an emergency differ from non-emergency patients in their consultation patterns and symptoms? A longitudinal data-linkage study in England
}

\author{
C Renzi ${ }^{*}, 1,2$, G Lyratzopoulos ${ }^{1,3}$, T Card ${ }^{4,5}$, TPC Chu 6 , U Macleod ${ }^{7}$ and B Rachet ${ }^{2}$
}

${ }^{1}$ Health Behaviour Research Centre, Department of Epidemiology and Public Health, University College London, WC1E 6BT London, UK; ${ }^{2}$ Cancer Survival Group, Department of Non-communicable Disease Epidemiology, London School of Hygiene and Tropical Medicine, WC1E 7HT London, UK; ${ }^{3}$ Cambridge Centre for Health Services Research, University of Cambridge, CB2 OSR Cambridge, UK; ${ }^{4}$ Division of Epidemiology and Public Health, University of Nottingham, NG5 1PB Nottingham, UK; ${ }^{5}$ Nottingham Digestive Diseases Centre Biomedical Research Unit, University of Nottingham, NG7 2UH Nottingham, UK; ${ }^{6}$ Division of Child Health, Obstetrics and Gynaecology, University of Nottingham, NG7 2UH Nottingham, UK and ${ }^{7}$ Hull York Medical School, University of Hull, HU6 7RX Kingston upon Hull, UK

Background: More than $20 \%$ of colorectal cancers are diagnosed following an emergency presentation. We aimed to examine pre-diagnostic primary-care consultations and related symptoms comparing patients diagnosed as emergencies with those diagnosed through non-emergency routes.

Methods: Cohort study of colorectal cancers diagnosed in England 2005 and 2006 using cancer registration data individually linked to primary-care data (CPRD/GPRD), allowing a detailed analysis of clinical information referring to the 5-year pre-diagnostic period.

Results: Emergency diagnosis occurred in 35\% and 15\% of the 1029 colon and 577 rectal cancers. 'Background' primary-care consultations ( $2-5$ years before diagnosis) were similar for either group. In the year before diagnosis, $>95 \%$ of emergency and non-emergency presenters had consulted their doctor, but emergency presenters had less frequently relevant symptoms (colon cancer: $48 \%$ vs 71\% $(P<0.001)$; rectal cancer: $49 \%$ vs 61\% $(P=0.043)$ ). 'Alarm' symptoms were recorded less frequently in emergency presenters (e.g., rectal bleeding: 9 vs $24 \%(P=0.002)$ ). However, about $1 / 5$ of emergency presenters ( 18 and $23 \%$ for colon and rectal cancers) had 'alarm' symptoms the year before diagnosis.

Conclusions: Emergency presenters have similar 'background' consultation history as non-emergency presenters. Their tumours seem associated with less typical symptoms, however opportunities for earlier diagnosis might be present in a fifth of them.

According to international data, between 14 and $33 \%$ of colorectal cancers are diagnosed as emergencies (Gunnarsson et al, 2014). Despite some recent progress, in England a diagnosis of cancer following an emergency presentation still occurs in as many as $22 \%$ of colorectal cancers, with significant socio-economic inequalities (NCIN, 2015). Emergency presenters are less often treated with curative intent (McArdle and Hole, 2004), even after controlling for stage at diagnosis (McPhail et al, 2013), and they have poorer survival (Elliss-Brookes et al, 2012; Downing et al, 2013). Moreover, emergency presentations are associated with worse patient-reported outcomes (Quality Health, 2014) and disruptions to hospital services (Goodyear et al, 2008). Reducing emergency

*Correspondence: Dr C Renzi; E-mail: c.renzi@ucl.ac.uk

Received 1 April 2016; revised 11 July 2016; accepted 18 July 2016; published online 18 August 2016

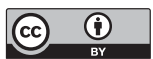


presentations could therefore lead to more efficient and appropriate use of health services, and substantially improve health outcomes.

However, studies examining potentially modifiable risk factors and circumstances surrounding emergency cancer diagnosis are limited (Mitchell et al, 2015a). Some studies have shown an increased risk of emergency colorectal cancer diagnosis for women (Abel et al, 2015), older (Mitchell et al, 2015a) and more deprived people (Raine et al, 2010; Mayor, 2012), but the findings are not always consistent. Few studies have examined colon and rectal cancers separately (McArdle and Hole, 2004; Rabeneck et al, 2006; Gunnarsson et al, 2013, 2014; Abel et al, 2015), even though these two cancer sites often have distinct clinical presentations and the prevalence of emergency diagnosis is markedly different (31\% for colon and 15\% for rectal cancers; Abel et al, 2015). Only very limited evidence is available on symptoms and health-care use before emergency cancer diagnosis. According to one Swedish study on colon cancer (Gunnarsson et al, 2014) and two UK studies on colorectal cancers diagnosed in London (Sheringham et al, 2014) and Exeter (Cleary et al, 2007) most patients have seen their doctor during the 6 months before diagnosis, often with non-specific symptoms. Case note reviews within clinical audits (Rubin et al, 2011), qualitative studies (Black et al, 2015) and patient surveys (Lyratzopoulos et al, 2012) have also provided some insights into potential opportunities to diagnose cancer earlier, but they are often limited by participation and recall bias, due to retrospective data collection after patients received a cancer diagnosis.

Some emergency diagnoses can be regarded as unavoidable, such as in the case of cancers with a sudden clinical presentation with minimal or no prior symptoms (Lyratzopoulos et al, 2014). Other cases are potentially avoidable and these include: (a) patients who, despite having symptoms, did not seek help promptly due to psycho-social factors or health-care system barriers (in this case public education and removing barriers to health care are necessary); (b) patients who sought help for symptoms, but opportunities were missed due to atypical symptoms, or deficiencies in investigations or other factors. The proportion of patients falling into each of the above categories is unknown.

In order to provide a population-level picture of symptomatic presentations during the months and years before the cancer diagnosis and to identify opportunities for reducing emergency diagnoses we used national cancer registration data individually linked to clinical data prospectively collected in primary care within the Clinical Practice Research Datalink (CPRD_previously GPRD). CPRD is a large database of anonymised primary-care records from over 600 general practices. It is validated and extensively used for epidemiological research and is considered to be representative of the UK population (Khan et al, 2010; Dregan et al, 2012; Tsang et al, 2013; Chu et al, 2015; Din et al, 2015). The database is particularly suited for the present study as it provides details on the medical history of patients, including prospectively recorded patient-level information on each episode of illness, symptom occurrences, all significant clinical contacts, diagnoses and abnormal test results.

The objectives of our study were to examine patterns of presentation in primary care with symptoms/signs potentially related to colon and rectal cancer during the years and months before the cancer diagnosis. In particular, we aimed to compare patients with a cancer diagnosis following an emergency presentation with patients diagnosed after non-emergency referrals, taking socio-demographic factors into account, in order to identify opportunities for reducing emergency presentations. This will be useful for providing evidence that can inform the development of interventions aimed at reducing emergency cancer diagnosis, and for improving quality of care and cancer outcomes.

\section{MATERIALS AND METHODS}

Study sample and data sources. We have conducted a cohort study using data from the population-based National Cancer Registry linked to CPRD/GPRD data for patients with an incident colon or rectal cancer (ICD10 codes C18 and C19-C20, respectively). We included cancers diagnosed in England in 2005 and 2006, as this represents the latest cohort with linked CPRD data available to us, providing information on signs and symptoms for up to 10 years before the cancer diagnosis (Ethics approval: ISAC-Protocol 08_031R; NHS Health Research Authority Confidentiality Advisory Group (PIAG 1-05(c)/2007)). The present study focused on the 5-year pre-diagnostic period, as an initial examination of consultation patterns going back to 10 years showed no relevant variations in consultation rates 5-10 years before the cancer diagnosis.

Inclusion criteria were age 25 years or older, no previous diagnosis of cancer at any site, at least 1 year of CPRD records before cancer diagnosis. Individuals with a previous cancer diagnosis were not included as they probably have different helpseeking behaviour and health-care use (due to increased cancer awareness and possibly regular follow-up visits) compared with primary-care patients overall. Doctors might also be more prone to consider cancer as a possible explanation for symptoms presented by these patients. This subgroup merits to be examined separately, but this was not possible in the present study due to small numbers.

The CPRD includes an 'up-to-standard' date, indicating when the data meet pre-defined quality criteria in over 80 variables. We included only records meeting these criteria in order to reduce the risk of missing or inaccurate data.

Of the 58359 incident colon and rectal cancer patients identified in the National Cancer Registry, 1922 patients were linked to CPRD (3.3\%). This was in line with expectations, considering that about half of all GP practices included in CPRD (covering 7\% of the population in England) participate in the data-linkage scheme. Non-participation in the linkage scheme is mostly due to nonresponse rather than active refusal. After applying the studyexclusion criteria a total of 1606 patients were included in the final study sample (Figure 1). On average, each GP practice contributed to 8 cancer patients over the total study period.

Variable definitions. Our outcome of interest was an emergency cancer diagnosis, defined according to the 'routes to diagnosis' algorithm based on several routine data sets and provided by NCIN (Elliss-Brookes et al, 2012; NCIN, 2013). In particular, an emergency diagnosis is defined as a diagnosis of cancer following presentation to an Accident and Emergency Unit, or following a GP emergency referral or following emergency pathways for in/outpatients (Elliss-Brookes et al, 2012; NCIN, 2013). Non-emergency cancer diagnoses include routine GP referrals, 2-week wait GP referrals (introduced in 2000 to allow GPs to refer suspected cancer patients urgently, so that they can see a specialist within 2 weeks), elective inpatient/outpatient and screening. For the purpose of our study focusing on emergency presentation, and similarly to previous research (McPhail et al, 2013), after an initial description of the different routes we have grouped patients into two categories: emergency and non-emergency cancer patients (the latter including all the non-emergency routes).

Our main explanatory variables were signs and symptoms recorded in primary care prior to the cancer diagnosis. On the basis of the published literature (Sheringham et al, 2014; Din et al, 2015) and guidelines (NICE Guidelines, 2015), we have operationally defined signs/symptoms potentially relevant for colorectal cancer. Our preliminary list has been reviewed by clinical experts and a final list has been compiled (Supplementary 

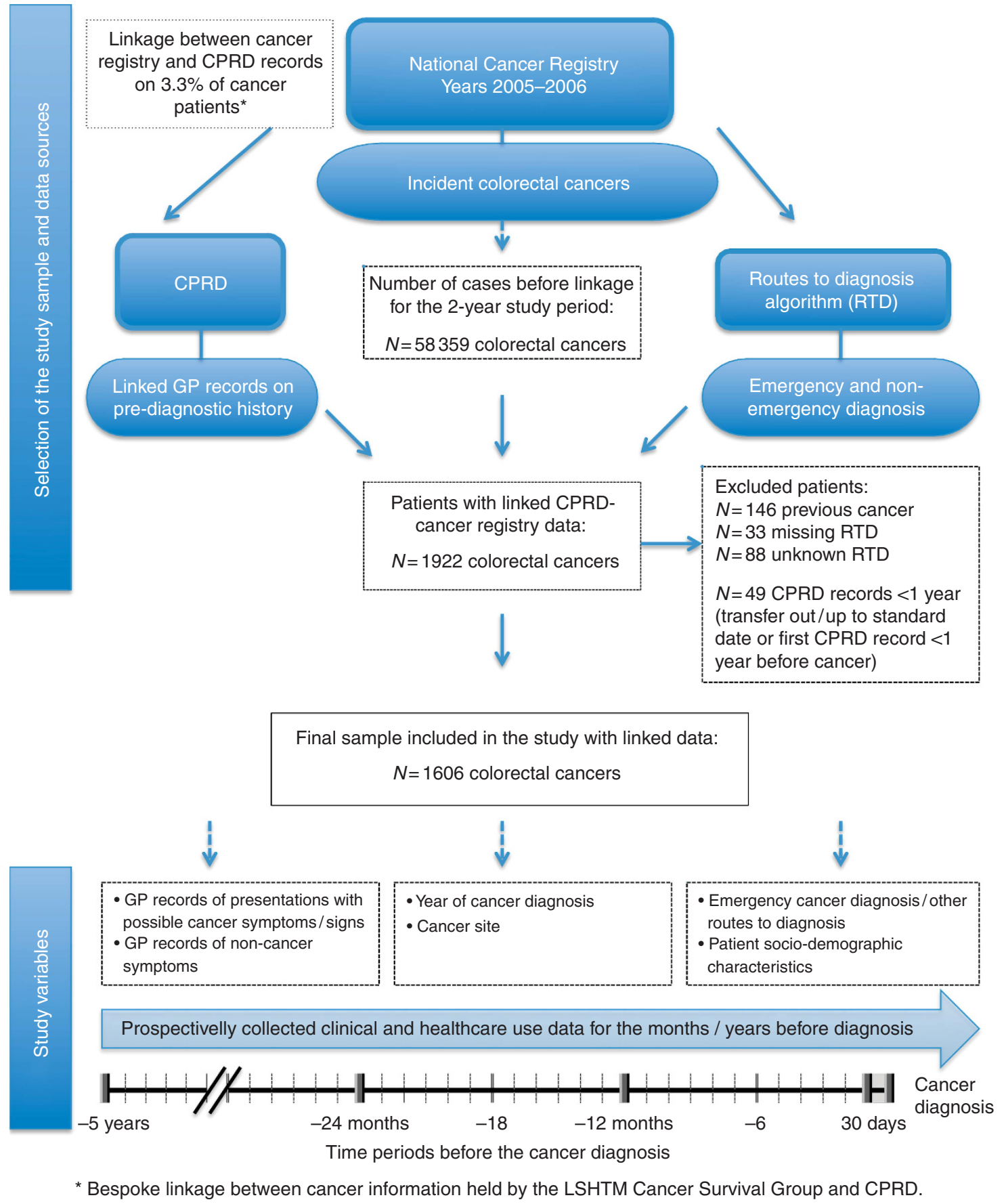

Figure 1. Study sample selection and data sources.

Material 1). Examples of relevant signs/symptoms are as follows: rectal bleeding, change in bowel habits, palpable rectal mass, irondeficiency anaemia, abdominal pain and weight loss. Read codes for relevant symptoms have been identified and applied to records in CPRD (Supplementary Material 1). The Read codes included in the final list are as comprehensive as possible, considering that different codes can be used for similar symptoms (e.g., 16 different codes were included for identifying patients with diarrhoea). It was based on codes used in previous studies (Sheringham et al, 2014) and further expanded following a detailed revision by clinical experts, as well as an examination of the data and the Read Code hierarchy (see Supplementary Material 2 for details on the development of the list of signs/symptoms).

In order to account for patient characteristics we also examined age, gender and deprivation, based on the income domain of the
Index of Multiple Deprivation for England (Department for Communities and Local Government, 2008, The English Indices of Deprivation, 2007 London).

Statistical analysis. We initially described the socio-demographic characteristics, number, type and timing of symptoms before the cancer diagnosis separately for patients with emergency and nonemergency presentation. Colon and rectal cancers were examined separately throughout.

We then examined predictors of emergency diagnosis in univariable analyses, and assessed significance using $\chi^{2}$-test (or test for trend for ordered categorical variables). To compare the median number of consultations for any reason $>24$ months before cancer diagnosis in emergency and non-emergency presenters we used the Wilcoxon rank-sum test. Similarly, 
consultations for any reason during the year before cancer diagnosis have been examined. As events occurring shortly before diagnosis might be related to the diagnostic episode itself, rather than represent opportunities for earlier diagnosis, the 30 days before diagnosis have been examined separately throughout.

We examined the proportions of patients with at least one relevant symptom and with each specific symptom in different time periods before the cancer diagnosis (Figure 1) and we compared these proportions between emergency and non-emergency presenters using $\chi^{2}$ statistics. Consultation rates with relevant symptoms over the 5-year time period have been calculated and divided in bi-monthly, six-monthly and yearly time periods, in order to examine changes in consultation rates over time. We have examined whether consultation rates with relevant symptoms significantly varied by emergency presentation status using Poisson regression. The models included age, sex and deprivation, and were fitted for each time period separately, focusing on the 6 months and the year before diagnosis, as well as 13-24 months and 25-36 months before diagnosis. Random effects were included to account for patient-level clustering of symptomatic presentations.

Finally, multivariable logistic regression was used for examining the risk of emergency diagnosis according to type and timing of symptoms, and taking into account the number of consultation for any reason during the year before diagnosis and socio-demographic characteristics. The final model included variables thought a priori to be potentially important explanatory variables based on previous evidence and clinical reasoning (i.e., socio-demographic factors and number of consultations), and the specific symptoms that were associated with emergency presentation at univariable analysis. As observations within GP practices are not independent (mean 8 observations per practice, range 1-26) robust standard errors were calculated.

Interactions between the variables included in the final model were examined (e.g., interaction between each symptom recorded the year before diagnosis and the same symptom in earlier time periods, and between symptoms and socio-demographic factors), but power was limited due to sparse data.

STATA14 software (Stata Corp, College Station, TX, USA) was used for statistical analyses.

\section{RESULTS}

Socio-demographic characteristics and emergency cancer diagnosis. Among the 1606 included patients 52\% of colon and 58\% of rectal cancer patients were men and the median age was 74 years (interquartile range (IQR) 65-81) and 73 years (IQR 63-80). The demographic characteristics of our study cohort were comparable with those of colorectal cancer patients in the 2005 and 2006 Cancer Registry not linked to CPRD. Among the study cohort, 35\% of colon and $15 \%$ of rectal cancer patients had an emergency cancer diagnosis.

An emergency diagnosis was more frequent in women $(P=0.04$ for both colon and rectal cancers), and older patients, particularly ages 80 years and above $(P=0.04$ for colon and $P=0.003$ for rectal cancers); it was also more frequent among socio-economic deprived patients for rectal cancers only $(P<0.001$; Table 1$)$.

Consultations for any reason before the cancer diagnosis. The great majority of the study cohort had primary-care information for the whole of the 5-year pre-diagnostic period, with only $2 \%$ of the cohort having primary-care records covering $<2$ years before diagnosis.

GP consultation rates per year for any reason during the time period 2-5 years before diagnosis were not significantly different between diagnostic routes, with $88 \%$ of both colon and rectal cancer patients having seen their GP at least once a year (Table 2); the median number of consultations per year was 5 (IQR 2-10) for non-emergency and emergency colon cancer patients; and 5 for both non-emergency and emergency rectal cancer patients (IQR 2-9 and 2-12, respectively). Consultations for any reason increased for all patients during the 13-24 months before diagnosis and even more so during the year before diagnosis. Specifically, as shown in Table 2, during the year before diagnosis consultations were significantly higher for non-emergency colon cancer patients (median 12; IQR 7-18) compared with emergency presenters (median 10; IQR 5-19). Non-emergency rectal cancer patients had fewer consultations during the year before diagnosis (median 9; IQR 5-13) compared with emergency presenters (median 12; IQR $6-20)$. Only a small minority of patients (2.4 and $3.1 \%$ of colon and rectal cancers, respectively) have never seen their GP during the year before diagnosis, with minimal differences between emergency and non-emergency presenters.

Consultations for relevant symptoms before the cancer diagnosis. The majority of patients had at least one consultation with a relevant symptom recorded during the year before diagnosis (80 and $84 \%$ among colon and rectal cancers, respectively; Table 3). However, the proportion of patients with at least one relevant symptom was significantly lower in emergency compared with non-emergency presenters, particularly when excluding the 30 days before diagnosis (colon: 48 vs $71 \%, P<0.001$; rectal cancers: $49 \%$ vs $61 \%, P=0.043$ ).

'Background' consultation rates with a potentially relevant symptom were very low and remained stable during the 5-year period up until $\sim 12-17$ months before diagnosis (Figure 2). For both colon and rectal cancer patients, consultation rates increased markedly during the year before diagnosis, particularly during the last 6 months, with no apparent differences by emergency presentation status. Using Poisson regression and controlling for socio-demographic variables showed that consultation rates during the year before diagnosis were not significantly different for emergency $v s$ non-emergency presenters (incidence rate ratio (IRR) for colon cancer $=0.86 ; 95 \%$ CI $0.7-1.1 ; P=0.182$; rectal cancer $=1.26 ; \quad 95 \% \quad$ CI $0.9-1.8 ; \quad P=0.210)$. However, when restricting to the last 6 months before diagnosis, emergency presenters with colon cancer had a significantly lower consultation rate $(\mathrm{IRR}=0.76 ; 95 \%$ CI $0.6-0.9 ; P=0.039)$.

Specific relevant symptoms before the cancer diagnosis. The potentially relevant symptoms/signs most frequently recorded during the year before diagnosis (excluding the 30 days) were abdominal pain $(25.1 \%)$, anaemia $(19.2 \%)$, diarrhoea $(9.9 \%)$ and rectal bleeding $(9.4 \%)$ among colon cancer patients, and rectal bleeding $(21.5 \%)$, change in bowel habits (11.6\%), diarrhoea (12\%) and abdominal pain $(9.4 \%)$ in rectal cancers patients (Table 3 ). However, symptoms were different according to emergency presentation status, particularly for colon cancers where 'red-flag symptoms' were more prevalent among non-emergency presenters compared with emergency presenters: anaemia (23.2 vs 11.9\%; $P<0.001)$, rectal bleeding $(12.6$ vs $3.6 \% ; P<0.001)$ and change in bowel habits (6.7 vs 3.3\%; $P=0.022)$. Among rectal cancer patients, only rectal bleeding was significantly more prevalent in non-emergency presenters ( 23.7 vs $9.2 \%$; $P=0.002$ ). Overall, $31.8 \%$ of colon cancer and $36.4 \%$ of rectal cancer patients had at least one of the above-mentioned 'red-flag' symptoms recorded between 30 days and 12 months pre-diagnosis. Non-emergency presenters had a higher prevalence of at least one red-flag symptom compared with emergency presenters (colon: 39.5 vs $17.5 \%$; $P<0.001$; rectal cancer: 38.8 vs $23 \%$; $P=0.005)$.

Among patients with at least one relevant symptom, $47 \%$ of colon and $43 \%$ of rectal cancer patients had multiple visits with the same symptom during the period between 30 days and 12 months pre-diagnosis, without statistical evidence for variation in this proportion by emergency presentation status (data not shown). 
Table 1. Diagnosis of colon or rectal cancer following EP by patients' socio-demographic characteristics (univariable analysis)

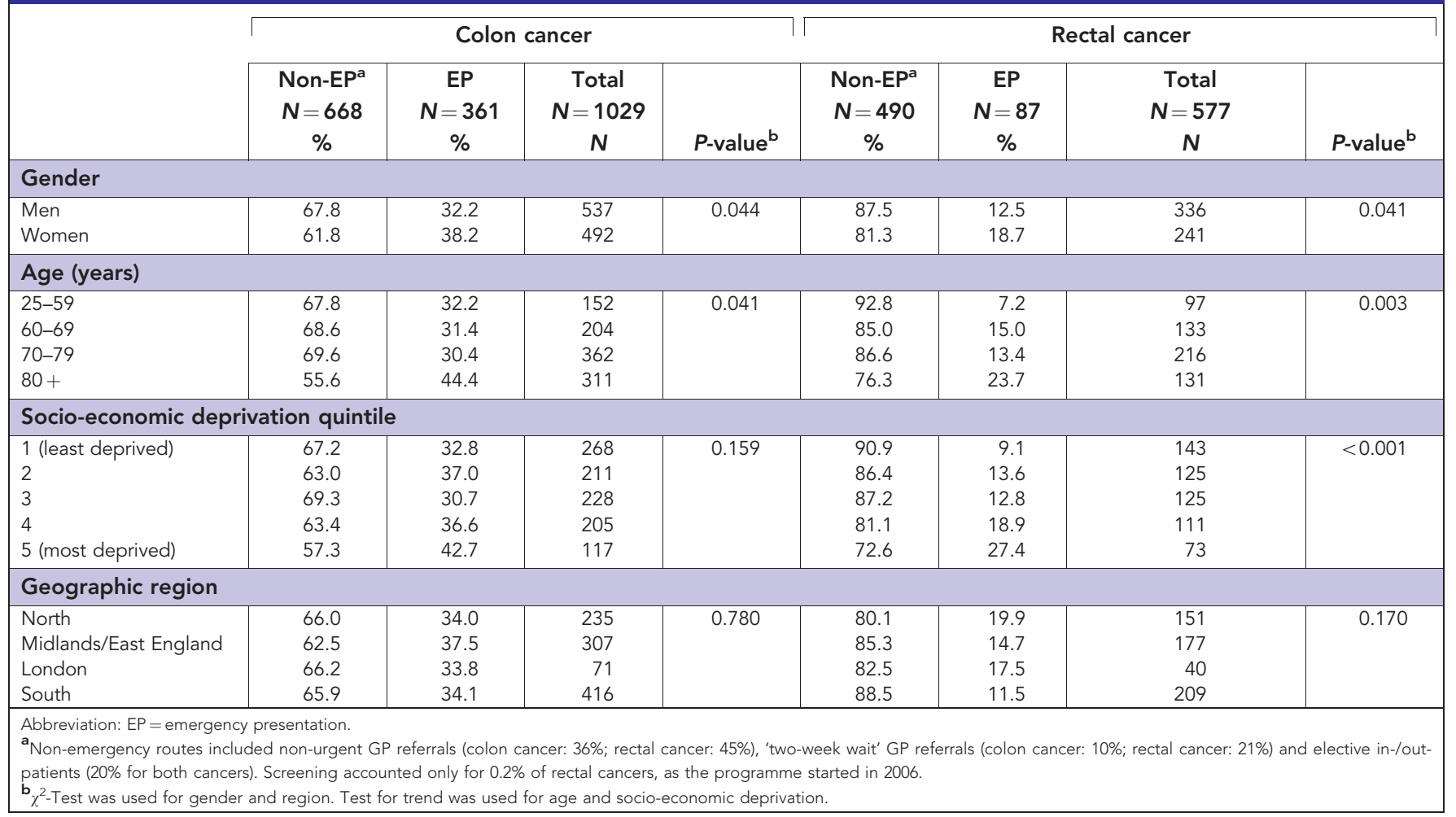

Table 2. GP consultations for any reason for patients diagnosed with colon or rectal cancer following EP vs non-EP

\begin{tabular}{|c|c|c|c|c|c|c|c|c|}
\hline & \multicolumn{4}{|c|}{ Colon cancer } & \multicolumn{4}{|c|}{ Rectal cancer } \\
\hline \multicolumn{9}{|c|}{ GP visits for any reason $25-60$ months pre-diagnosis (number of visits per year) } \\
\hline $\begin{array}{c}\text { Median (IQR) } \\
0 \text { visits } \\
1-2 \text { visits } \\
3-4 \text { visits } \\
5-9 \text { visits } \\
10+\text { visits }\end{array}$ & $\begin{array}{l}12.1 \\
18.8 \\
16.3 \\
28.8 \\
24.1 \\
\end{array}$ & $\begin{array}{c}5(2-10) \\
12.9 \\
17.1 \\
17.5 \\
28.0 \\
24.6 \\
\end{array}$ & $\begin{array}{c}5(2-10) \\
10.5 \\
21.9 \\
14.1 \\
30.2 \\
23.3 \\
\end{array}$ & $\begin{array}{l}0.739 \\
0.756\end{array}$ & $\begin{array}{l}12.1 \\
21.5 \\
15.9 \\
27.7 \\
22.7 \\
\end{array}$ & $\begin{array}{c}5(2-9) \\
12.7 \\
21.0 \\
16.1 \\
29.2 \\
21.0 \\
\end{array}$ & $\begin{array}{c}5(2-12) \\
9.2 \\
24.1 \\
14.9 \\
19.5 \\
32.2 \\
\end{array}$ & $\begin{array}{l}0.226 \\
0.124\end{array}$ \\
\hline \multicolumn{9}{|c|}{ GP visits for any reason 13-24 months pre-diagnosis (number of visits per year) } \\
\hline $\begin{array}{c}\text { Median (IQR) } \\
0 \text { visits } \\
1-2 \text { visits } \\
3-4 \text { visits } \\
5-9 \text { visits } \\
10+\text { visits }\end{array}$ & $\begin{array}{r}6.0 \\
13.5 \\
12.4 \\
24.7 \\
43.4 \\
\end{array}$ & $\begin{array}{c}8(3-14) \\
9.1 \\
14.7 \\
13.3 \\
24.4 \\
38.5\end{array}$ & $\begin{array}{c}7(3-13) \\
7.1 \\
13.9 \\
12.7 \\
24.6 \\
41.7\end{array}$ & $\begin{array}{l}0.038 \\
0.056\end{array}$ & $\begin{array}{r}8.4 \\
18.2 \\
12.2 \\
31.8 \\
29.4 \\
\end{array}$ & $\begin{array}{c}6(2-11) \\
6.9 \\
6.9 \\
12.6 \\
28.7 \\
44.8\end{array}$ & $\begin{array}{c}9(4-15) \\
8.2 \\
16.5 \\
12.3 \\
31.4 \\
31.7\end{array}$ & $\begin{array}{l}0.002 \\
0.002\end{array}$ \\
\hline $\begin{array}{c}\text { Median (IQR) } \\
0 \text { visits } \\
1-2 \text { visits } \\
3-4 \text { visits } \\
5-9 \text { visits } \\
10+\text { visits }\end{array}$ & $\begin{array}{r}2.4 \\
5.3 \\
7.2 \\
26.3 \\
58.8 \\
\end{array}$ & $\begin{array}{c}12(7-18) \\
2.1 \\
3.9 \\
6.3 \\
26.8 \\
60.9 \\
\end{array}$ & $\begin{array}{c}10(5-19) \\
3.1 \\
7.8 \\
8.9 \\
25.5 \\
54.9 \\
\end{array}$ & $\begin{array}{l}0.041 \\
0.008\end{array}$ & $\begin{array}{r}3.1 \\
9.2 \\
11.3 \\
29.3 \\
47.1 \\
\end{array}$ & $\begin{array}{c}9(5-13) \\
3.1 \\
9.0 \\
12.2 \\
30.6 \\
45.1 \\
\end{array}$ & $\begin{array}{c}12(6-20) \\
3.5 \\
10.3 \\
5.8 \\
21.8 \\
58.6 \\
\end{array}$ & $\begin{array}{l}0.010 \\
0.068\end{array}$ \\
\hline
\end{tabular}

Examining potentially relevant symptoms recorded in more distant years (i.e., between 25-60 months pre-diagnosis) has shown that emergency rectal cancer patients had more frequently a past record of anaemia (8.1 vs 2.0\%; $P=0.002$ ) and change in bowel habits (2.3 vs $0.4 \% ; P=0.050)$ compared with non-emergency presenters. Among colon cancer patients, emergency presenters had less frequently a past record of rectal bleeding (1.7 vs 3.9\%; $P=0.049)$ than non-emergency presenters. Overall, the prevalence of at least one red-flag symptom was much lower during the more distant time periods compared with the year before diagnosis 

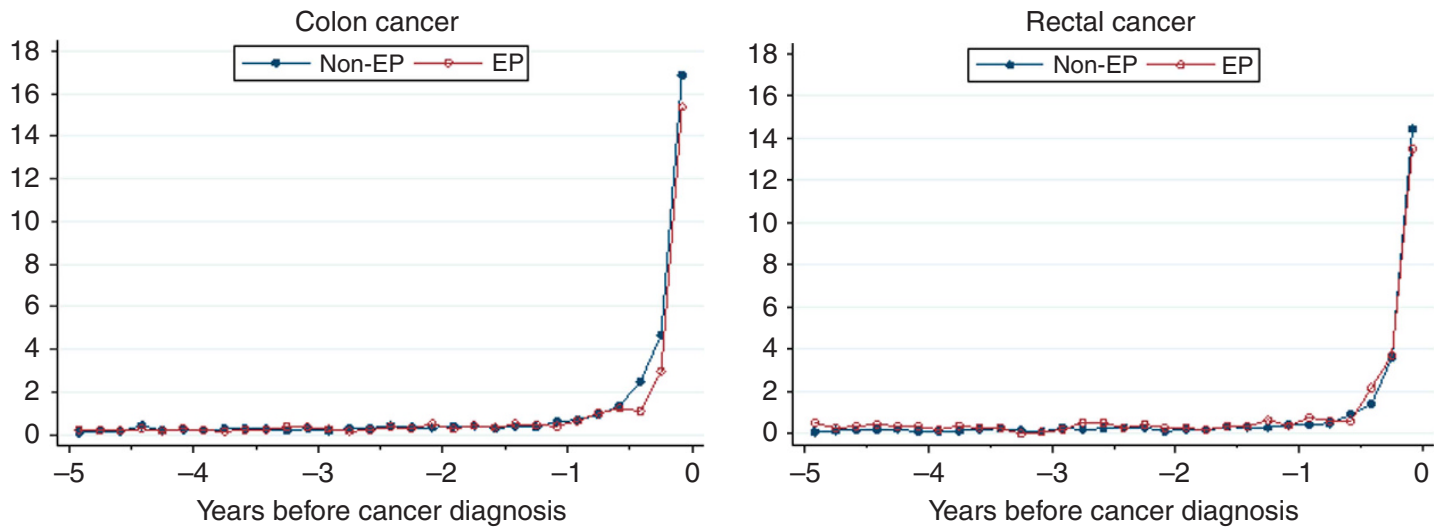

Figure 2. Rates of consultations with relevant symptoms for emergency (EP) and non-emergency (non-EP) presenters: bi-monthly rates (per 100 person-years).

(e.g., 5.9 and $4.7 \%$ among colon and rectal cancers, respectively, 13-24 months before diagnosis) without apparent differences by emergency presentation status.

Multivariable analysis examining the effect of symptomatic presentations and socio-demographic factors on emergency cancer diagnosis. Multivariable logistic regression analysis, including socio-demographic factors and relevant symptoms into the model, has shown that in the period from 30 days to 12 months pre-diagnosis the risk of emergency colon cancer diagnosis was significantly lower for patients with a record of anaemia $(\mathrm{OR}=0.38 ; 95 \%$ CI $0.3-0.6)$, change in bowel habits $(\mathrm{OR}=0.47$; 95\% CI $0.3-0.9)$ or rectal bleeding ( $\mathrm{OR}=0.22$; 95\% CI $0.1-0.4$; Table 4). On the other hand, emergency diagnosis was more likely in women $(\mathrm{OR}=1.37 ; 95 \% \mathrm{CI} 1.0-1.8)$ and people aged 80 years and older $(\mathrm{OR}=1.84 ; 95 \% \mathrm{CI} 1.2-2.7)$, independently of symptom history. For rectal cancers, only rectal bleeding during the year before diagnosis was associated with a lower risk of emergency presentation $(\mathrm{OR}=0.25 ; 95 \%$ CI $0.1-0.6)$. Socio-economic deprivation was associated with a higher risk of emergency presentation for rectal cancer, independently of symptoms (e.g., most deprived category $\mathrm{OR}=3.47 ; 95 \% \mathrm{CI} 1.5-8.0)$. Increasing number of consultations for any reason during the year before diagnosis somewhat increased the risk of emergency presentation for rectal cancer $(\mathrm{OR}=1.03 ; 95 \%$ CI $1.0-1.1)$. This was also confirmed after excluding outliers, that is, patients with a very high number of consultations (upper 5th percentile, corresponding to $>32$ consultations during the year before diagnosis; data not shown). There was some indication that change in bowel habits $(\mathrm{OR}=12.0 ; 95 \%$ CI $1.6-92.1)$ and anaemia $(\mathrm{OR}=2.67 ; 95 \%$ CI $0.8-8.9)$ recorded 25-60 months pre-diagnosis might increase the risk of emergency rectal cancer but confidence intervals were wide, reflecting the small number of individuals with such records.

\section{DISCUSSION}

Main findings. Linked cancer registration and primary-care data allowed for a detailed description of clinical presentations in primary care before a cancer diagnosis, comparing patients diagnosed as an emergency with those diagnosed through non-emergency routes. The longitudinal data have shown that consultation patterns between 12 months and up to 5 years pre-diagnosis were very similar in emergency and non-emergency presenters. Consultation rates increased significantly in the last months before diagnosis independently of the diagnostic route. Emergency presenters are not a uniform category and they can be divided into different groups according to their consultation history. Only a very small minority of emergency presenters have never consulted for any reason during the year before diagnosis. However, less than half of emergency presenters have clinical records of relevant cancer symptoms, which is significantly lower than among non-emergency presenters. Nevertheless, approximately a fifth of emergency presenters had typical 'alarm' symptoms and $16 \%$ had 3 or more consultations with relevant symptoms, suggesting possible opportunities for earlier diagnosis.

Comparison with other studies and possible explanations for our findings. Our findings are in line with previous studies showing that most emergency presenters have primary-care consultations during the months before the cancer diagnosis (Cleary et al, 2007; Gunnarsson et al, 2014; Sheringham et al, 2014). Our results are also in agreement with a study based on direct record reviews reporting that $60 \%$ of emergency colorectal cancer patients had relevant symptoms 1 month or more before diagnosis (Cleary et al, 2007).

Abdominal pain and rectal bleeding are the most frequent symptoms among colon and rectal cancer patients, respectively, (Hamilton et al, 2013) and similarly to previous research, we found a lower risk of emergency presentation for patients with rectal bleeding, a well-recognised symptom of colorectal cancer (Cleary et al, 2007; Gunnarsson et al, 2014; Sheringham et al, 2014). Earlier research highlighted an increased risk of emergency diagnosis in case of abdominal pain and constipation (Sheringham et al, 2014), diarrhoea and weight loss (Cleary et al, 2007). Concordantly, we found that these symptoms/signs were all associated with emergency diagnosis, but only when focusing on the last 30 days before diagnosis. These symptoms/signs can be an indication of progression towards occlusion, which may explain their higher prevalence among emergency presenters shortly before diagnosis.

Anaemia and change in bowel habits, typical red-flag symptoms generally leading to prompt investigations, were also associated with a lower risk of emergency colon cancer diagnosis. Anaemia and change in bowel habits recorded $2-5$ years pre-diagnosis might increase the risk of emergency presentation, but sparse data limited our analyses. These sign/symptoms might have been initially dismissed as benign and subsequently neglected by patients and/or doctors, as suggested by previous research (Mitchell et al, 2015b; Renzi et al, 2016).

Importantly, our study has highlighted that during the year before diagnosis one in five emergency presenters had at least one red-flag symptom, suggesting opportunities for earlier diagnosis in these cases. Opportunities are probably even more prevalent, considering that symptoms are likely to be under-recorded, as suggested by the fact that one out of three non-emergency presenters had no relevant symptom recorded the year before diagnosis. 
Table 3. GP consulations with relevant symptoms for patients diagnosed with colon or rectal cancer following EP and non-EP by time before diagnosis

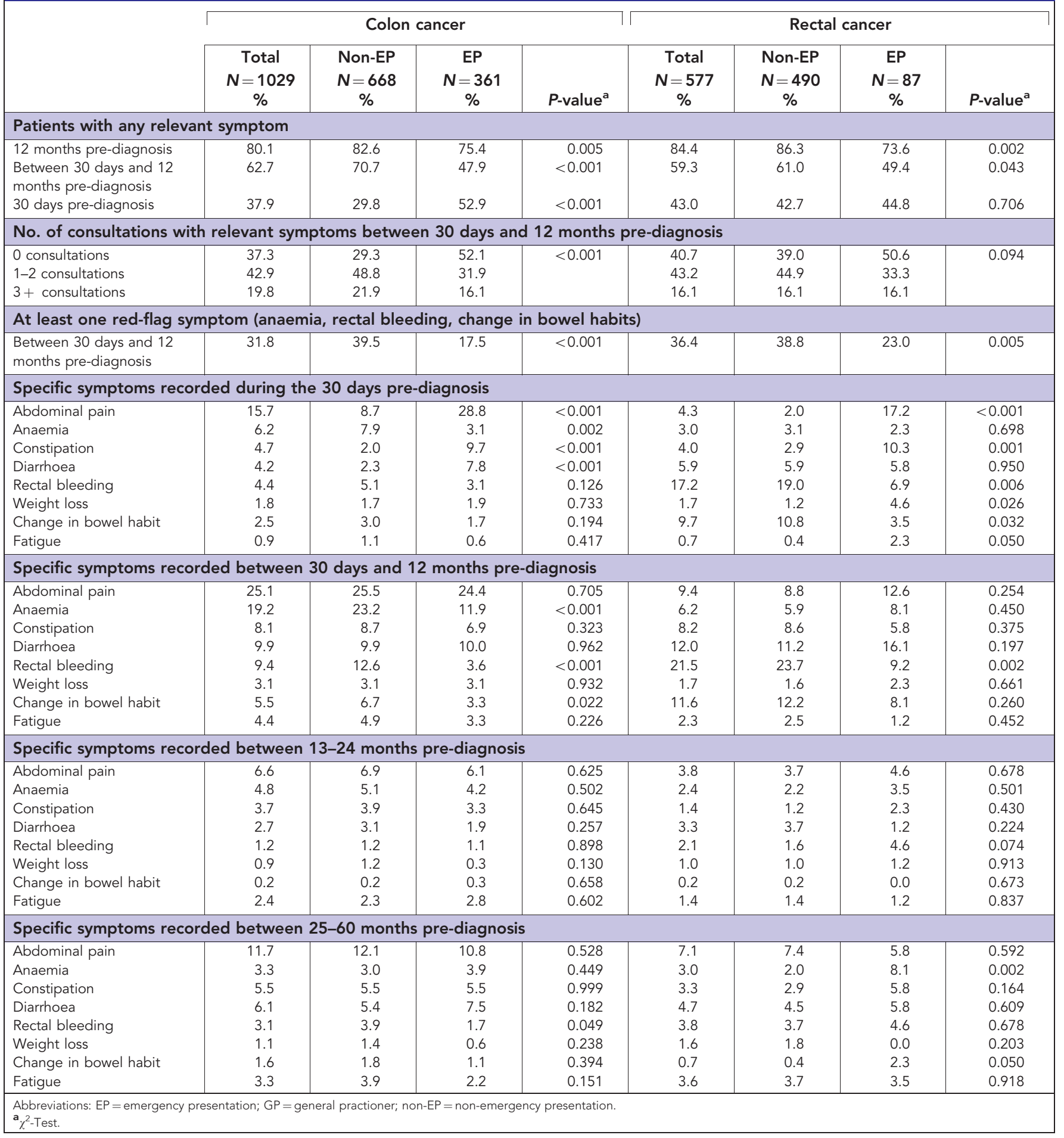

On the basis of international data, missed opportunities can occur in 1 out of 3 colorectal cancer patients, with older age, comorbidities and belonging to ethnic minority groups increasing the risk (Singh et al, 2009). Multiple factors are often implicated, including patient, doctor and health-care system factor (Lyratzopoulos et al, 2015).

We found that between 16 and $22 \%$ of colon and rectal cancer patients had three or more consultations with relevant symptoms during the year before diagnosis, which is consistent with UK audit data (Rubin et al, 2011). Our study has highlighted that consultation rates overall and consultations with relevant symptoms increased substantially during the months before diagnosis among emergency and non-emergency presenters. In the case of rectal cancers the risk of emergency presentation increased with increasing number of consultations for any reason. This is in contrast with previous studies (Sheringham et al, 2014), but differences between colon and rectal cancers, and changes in the patterns of symptoms during the last 30 days before diagnosis were previously not taken into account. 
Table 4. Multivariable logistic regression OR for colon and rectal cancer diagnosed after EP compared with non-EP taking into account patient socio-demographic characteristics, number of GP consultations for any reason the year before diagnosis (excluding 30 days) and symptoms recorded in primary care $(N=1606)$

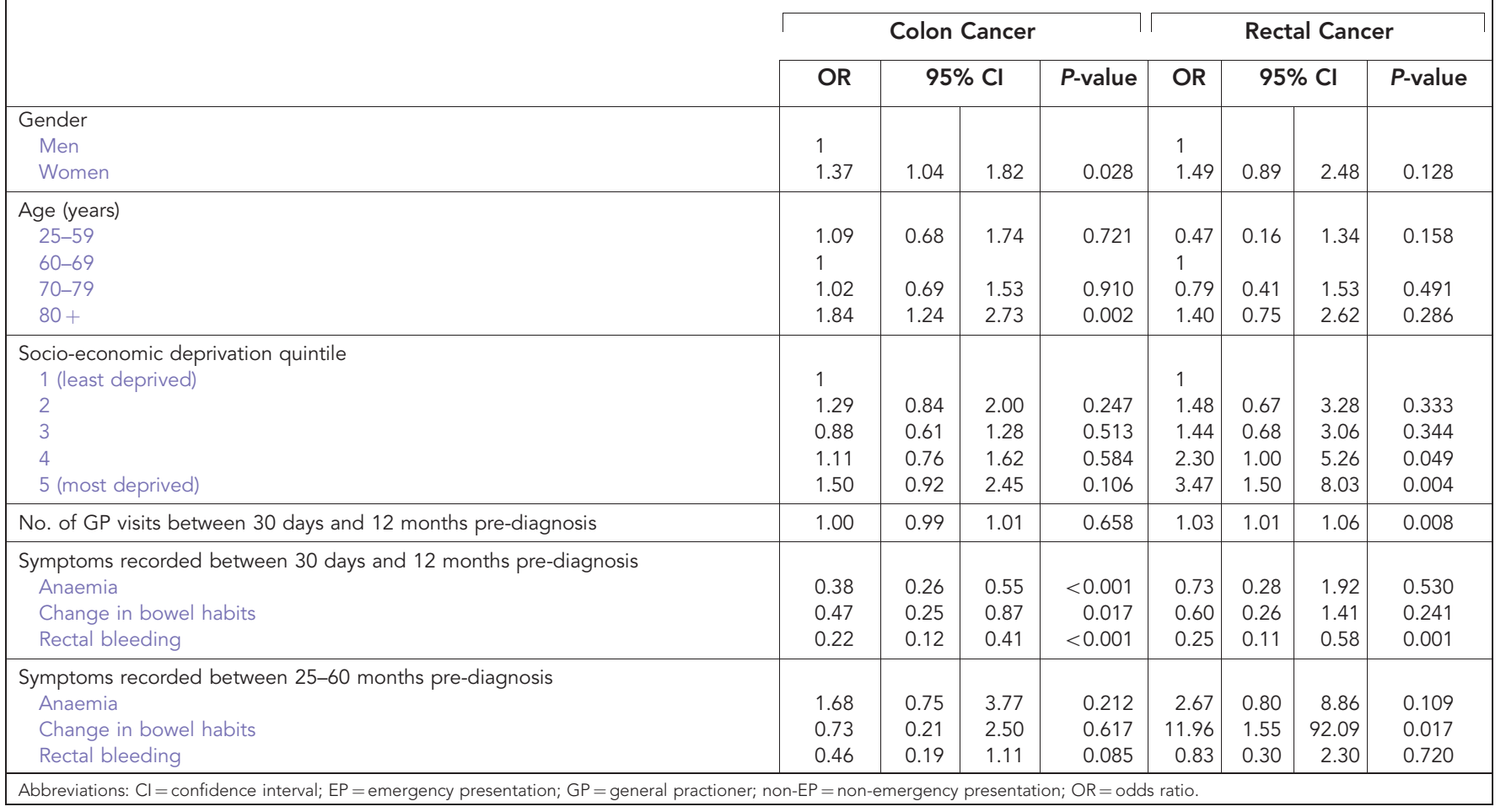

Our study has shown that in some cases despite specific symptoms, cancer was only diagnosed after emergency presentation, and this more likely occurred in some subgroups. Women, older and more deprived individuals have been previously shown to be at higher risk of emergency diagnosis (Raine et al, 2010; Mayor, 2012; Abel et al, 2015; Mitchell et al, 2015a), and our data indicate that these subgroups are at higher risk independently of symptomatic presentations. Further research is warranted to understand the role played by patient factors (e.g., missed follow-up visits), health-care factors (e.g., delays in diagnostic work-up, previous borderline/normal test results), as well as clinical and tumour factors complicating the diagnosis (comorbidities, proximal cancers). For example, indepth quantitative and qualitative studies would be necessary examining the role of comorbidities (Barnett et al, 2012; Mitchell et al, 2015b), their effect on patients' interpretation and reporting of cancer symptoms, as well as their effect on doctors' decision-making regarding differential diagnosis, referrals and testing.

The bowel cancer screening programme started in 2006 in England and limited evidence is available on a possible positive effect of screening and other early diagnosis/cancer awareness initiatives (NICE Guidelines, 2015; Be Clear on Cancer, 2016) on emergency presentations (Goodyear et al, 2008; Mansouri et al, 2015). Due to socio-economic differences in screening uptake (von Wagner et al, 2011), inequalities in emergency presentations and cancer outcomes may, however, persist. Dedicating particular attention to higher-risk groups will therefore remain paramount.

Strengths and limitations. The strengths of the study include the use of prospectively recorded population-based data comparing emergency and non-emergency cancer diagnoses defined according to validated methodologies (Elliss-Brookes et al, 2012; NCIN, 2013). Thanks to the high quality of the data sources, missing information on routes to diagnosis and sociodemographic characteristics were negligible. Moreover, our study cohort was comparable in terms of demographic characteristics to colorectal cancer patients in the Cancer Registry not linked to CPRD. Our study provided specific clinical insights for colon and rectal cancers regarding the pre-diagnostic period. By simultaneously evaluating the role of symptomatic presentations and patient characteristics we identified subgroups at higher risk of missed opportunities and emergency diagnosis, who could benefit from increased clinical and public health efforts. The study demonstrates the usefulness of linked cancer registration and primary-care data (such as CPRD) for early diagnosis research.

Our study will need to be extended to more recent cohorts of cancer patients with individually linked primary-care data. However, although some changes occurred since the study period in guidelines, clinical practice and patient awareness of symptoms (Moffat et al, 2015), the natural history of colorectal cancer and the disease processes determining the occurrence of signs and symptom will not have changed. It is also noteworthy that emergency presentations have remained stable over recent years for rectal cancers with a slight decrease for colon cancers (Abel et al, 2015); moreover, sociodemographic inequalities in emergency presentations (Abel et al, 2015) and cancer survival (Ellis et al, 2012) are still relevant (NCIN, 2015). We have performed sensitivity analyses evaluating whether our results differed for patients diagnosed in 2005 and 2006, which showed that the overall findings were not affected by the year of diagnosis in our sample.

Our results have to be interpreted with caution as the examined symptomatic presentations are based on clinical records and do not fully reflect all symptoms experienced by patients. However, this can be assumed to apply equally to emergency and non-emergency presenters. Moreover, clinical data were recorded prospectively by $>200$ GP practices before the cancer diagnosis, and emergency 
and non-emergency patients had similar records regarding their background consultation history.

Although routine data sources may contain inaccuracies, the validity of diagnostic coding and consultation rates in CPRD has been extensively confirmed (Khan et al, 2010; Dregan et al, 2012). CPRD are electronic versions of case notes and therefore include data reported by patients and considered relevant by doctors. It should be noted that sometimes doctors record clinical information only in free-text format rather than READ codes (Price et al, 2016). We did not have access to freetext information, which might have led to an underestimation of symptoms. Interviews with patients/doctors could verify the validity and improve accuracy, but this is beyond the purpose of the present work. Similarly, we lacked data on patient experience which can provide important insights. The possibility of linking CPRD records to patient experience data is an area that would merit future consideration in order to overcome this limitation.

Implications of findings. This study has shown that emergency presenters have similar 'background' consultation history as nonemergency presenters and their consultation rates increase markedly the year before diagnosis. Even though their tumours seem associated with less typical symptoms, opportunities for earlier diagnosis might be present in a fifth of them. In order to reduce emergency presentations, multi-disciplinary system-wide approaches are needed (Lyratzopoulos et al, 2015) addressing critical points along the diagnostic process, as well as targeting different patient subgroups (Borowski et al, 2016). More specifically, our findings underscore the importance of dedicating particular attention to patients consulting more frequently than usual, even if their symptoms are not immediately suggestive of cancer. In these cases, and in particular if patients belong to categories at higher risk of emergency diagnosis, such as the elderly, women and socio-economically deprived individuals, a variety of approaches could be employed. Specifically, these can include more pro-active and systematic symptom elicitation (Birt et al, 2014; McLachlan et al, 2015) and symptom monitoring ensuring a holistic approach (Mitchell et al, 2015b), possibly with the support of alternative health-care providers. Considering that a typical GP will only have around $10 \mathrm{~min}$ per appointment (Independent Cancer Taskforce, 2015), a specifically trained nurse could support the GP during the initial diagnostic phases and for subsequent follow-up visits and safety-netting. Pre-booked followup visits could be particularly useful for patients belonging to higher-risk groups (Mitchell et al, 2015b). Moreover, closer interaction and easier access to specialist advice for GPs would be important, in addition to the development of multi-disciplinary diagnostic centres (Independent Cancer Taskforce, 2015). Clinicians and public education campaigns should not only emphasise the importance of discussing symptoms with the doctor when they first appear, but also encourage and support subsequent monitoring of symptoms facilitating prompt re-evaluation if symptoms do not improve.

Regarding the subgroup of patients presenting with relevant symptoms, more systematic use of safety-netting, and prompt specialist referrals and diagnostic investigations would help to seize the opportunities for earlier diagnosis.

Reducing emergency presentations will allow more efficient and appropriate use of health services, improve patient experience of care and increase survival for cancer patients.

\section{ACKNOWLEDGEMENTS}

We thank the lay members and patient representatives who took part in discussing the initial phases of the project providing their insights into areas that need to be addressed in order to reduce emergency presentations. Funding for this work was provided by the Early Diagnosis Advisory Group (EDAG), Cancer Research UK [C48748/A18667]. GL is supported by Cancer Research UK Clinician Advanced Scientist Fellowship A18180.

\section{CONFLICT OF INTEREST}

The authors declare no conflict of interest.

\section{REFERENCES}

Abel G, Shelton J, Johnson S, Elliss-Brookes L, Lyratzopoulos G (2015) Cancer-specific variation in emergency presentation by sex, age and deprivation across 27 common and rarer cancers. $\mathrm{Br}$ J Cancer 112(Suppl 1): S129-S136.

Barnett K, Mercer SW, Norbury M, Watt G, Wyke S, Guthrie B (2012) Epidemiology of multimorbidity and implications for health care, research, and medical education: a cross-sectional study. Lancet 380: $37-43$.

Be Clear on Cancer (2016) Available at: http://www.cancerresearchuk.org/ health-professional/early-diagnosis-activities/be-clear-on-cancer. Accessed on 25 January 2016.

Birt L, Hall N, Emery J, Banks J, Mills K, Johnson M, Hamilton W, Walter FM (2014) Responding to symptoms suggestive of lung cancer: a qualitative interview study. BMJ Open Respir Res 1: e000067.

Black G, Sheringham J, Spencer-Hughes V, Ridge M, Lyons M, Williams C, Fulop N, Pritchard-Jones K (2015) Patients' experiences of cancer diagnosis as a result of an emergency presentation: a qualitative study. PloS One 10: e0135027.

Borowski DW, Cawkwell S, Zaidi SM, Toward M, Maguire N, Gill TS (2016) Primary care referral practice, variability and socio-economic deprivation in colorectal cancer. Colorectal Dis; e-pub ahead of print 25 April 2016; doi:10.1111/codi.13360.

Chu TP, Shah A, Walker D, Coleman MP (2015) Pattern of symptoms and signs of primary intracranial tumours in children and young adults: a record linkage study. Arch Dis Child 100: 1115-1122.

Cleary J, Peters TJ, Sharp D, Hamilton W (2007) Clinical features of colorectal cancer before emergency presentation: a population-based case-control study. Fam Pract 24: 3-6.

Din NU, Ukoumunne OC, Rubin G, Hamilton W, Carter B, Stapley S, Neal RD (2015) Age and gender variations in cancer diagnostic intervals in 15 cancers: analysis of data from the UK Clinical Practice Research Datalink. PloS One 10: e0127717.

Downing A, Aravani A, Macleod U, Oliver S, Finan PJ, Thomas JD, Quirke P, Wilkinson JR, Morris EJ (2013) Early mortality from colorectal cancer in England: a retrospective observational study of the factors associated with death in the first year after diagnosis. Br J Cancer 108: 681-685.

Dregan A, Moller H, Murray-Thomas T, Gulliford MC (2012) Validity of cancer diagnosis in a primary care database compared with linked cancer registrations in England. Population-based cohort study. Cancer Epidemiol 36: 425-429.

Ellis L, Coleman MP, Rachet B (2012) How many deaths would be avoidable if socioeconomic inequalities in cancer survival in England were eliminated? A national population-based study, 1996-2006. Eur J Cancer 48: $270-278$.

Elliss-Brookes L, McPhail S, Ives A, Greenslade M, Shelton J, Hiom S, Richards M (2012) Routes to diagnosis for cancer-determining the patient journey using multiple routine data sets. $\mathrm{Br} J$ Cancer 107: $1220-1226$.

Goodyear SJ, Leung E, Menon A, Pedamallu S, Williams N, Wong LS (2008) The effects of population-based faecal occult blood test screening upon emergency colorectal cancer admissions in Coventry and north Warwickshire. Gut 57: 218-222.

Gunnarsson H, Ekholm A, Olsson LI (2013) Emergency presentation and socioeconomic status in colon cancer. Eur J Surg Oncol 39: 831-836.

Gunnarsson H, Jennische K, Forssell S, Granstrom J, Jestin P, Ekholm A, Olsson LI (2014) Heterogeneity of colon cancer patients reported as emergencies. World J Surg 38: 1819-1826.

Hamilton W, Coleman MG, Rubin G (2013) Colorectal cancer. BMJ 346: f3172. 
Independent Cancer Taskforce (2015) Achieving world-class cancer outcomes: a strategy for England 2015-2020. Available at: http:// www.cancerresearchuk.org/sites/default/files/achieving_worldclass_cancer_outcomes_-_a_strategy_for_england_2015-2020.pdf. Accessed on 30 March 2016.

Khan NF, Harrison SE, Rose PW (2010) Validity of diagnostic coding within the General Practice Research Database: a systematic review. Br J Gen Pract 60: e128-e136.

Lyratzopoulos G, Neal RD, Barbiere JM, Rubin GP, Abel GA (2012) Variation in number of general practitioner consultations before hospital referral for cancer: findings from the 2010 National Cancer Patient Experience Survey in England. Lancet Oncol 13: 353-365.

Lyratzopoulos G, Saunders CL, Abel GA (2014) Are emergency diagnoses of cancer avoidable? A proposed taxonomy to motivate study design and support service improvement. Future Oncol 10: 1329-1333.

Lyratzopoulos G, Vedsted P, Singh H (2015) Understanding missed opportunities for more timely diagnosis of cancer in symptomatic patients after presentation. $\mathrm{Br} J$ Cancer 112(Suppl): S84-S91.

Mansouri D, McMillan D, Crearie C, Morrison D, Crighton E, Horgan P (2015) Temporal trends in mode, site and stage of presentation with the introduction of colorectal cancer screening: a decade of experience from the West of Scotland. Br J Cancer 113: 556-561.

Mayor S (2012) One in four cases of bowel cancer in England are diagnosed only after emergency admission. BMJ 345: e7117.

McArdle CS, Hole DJ (2004) Emergency presentation of colorectal cancer is associated with poor 5-year survival. Br J Surg 91: 605-609.

McLachlan S, Mansell G, Sanders T, Yardley S, van der Windt D, Brindle L, Chew-Graham C, Little P (2015) Symptom perceptions and help-seeking behaviour prior to lung and colorectal cancer diagnoses: a qualitative study. Fam Pract 32: 568-577.

McPhail S, Elliss-Brookes L, Shelton J, Ives A, Greenslade M, Vernon S, Morris EJ, Richards M (2013) Emergency presentation of cancer and short-term mortality. Br J Cancer 109: 2027-2034.

Mitchell E, Pickwell-Smith B, Macleod U (2015a) Risk factors for emergency presentation with lung and colorectal cancers: a systematic review. BMJ Open 5: e006965.

Mitchell E, Rubin G, Merriman L, Macleod U (2015b) The role of primary care in cancer diagnosis via emergency presentation: qualitative synthesis of significant event reports. Br J Cancer 112(Suppl): S50-S56.

Moffat J, Bentley A, Ironmonger L, Boughey A, Radford G, Duffy S (2015) The impact of national cancer awareness campaigns for bowel and lung cancer symptoms on sociodemographic inequalities in immediate key symptom awareness and GP attendances. Br J Cancer 112(Suppl 1): S14-S21.

NCIN (2013) Routes to Diagnosis: Exploring Emergency Presentations. Public Health England.

NCIN (2015) Routes to diagnosis 2006-2013 workbook. Public Health England.
NICE Guidelines (2015) Guidelines [NG12]. Suspected cancer: recognition and referral. Available at: http://www.nice.org.uk/guidance/NG12/chapter/1Recommendations-organised-by-site-of-cancer. Accessed on 15 February 2016.

Price SJ, Stapley SA, Shephard E, Barraclough K, Hamilton WT (2016) Is omission of free text records a possible source of data loss and bias in Clinical Practice Research Datalink studies? A case-control study. BMJ Open 6: e011664.

Quality Health (2014) Cancer Patient Experience Survey 2014 National Report. Available at: https://www.quality-health.co.uk/surveys/nationalcancer-patient-experience-survey. Accessed on 15 March 2016.

Rabeneck L, Paszat LF, Li C (2006) Risk factors for obstruction, perforation, or emergency admission at presentation in patients with colorectal cancer: a population-based study. Am J Gastroenterol 101: 1098-1103.

Raine R, Wong W, Scholes S, Ashton C, Obichere A, Ambler G (2010) Social variations in access to hospital care for patients with colorectal, breast, and lung cancer between 1999 and 2006: retrospective analysis of hospital episode statistics. BMJ 340: b5479.

Renzi C, Whitaker KL, Winstanley K, Cromme S, Wardle J (2016) Unintended consequences of an 'all-clear' diagnosis for potential cancer symptoms: a nested qualitative interview study with primary care patients. Br J Gen Pract 66: e158-e170.

Rubin G, McPhail S, Elliot K (2011) National Audit of Cancer Diagnosis in Primary Care 2011. Available at: http://www.cancerresearchuk.org/cancerinfo/spotcancerearly/naedi/AboutNAEDI/Optimisingclinicalpracticeandsystems/. Accessed on 15 February 2016.

Sheringham JR, Georghiou T, Chitnis XA, Bardsley M (2014) Comparing primary and secondary health-care use between diagnostic routes before a colorectal cancer diagnosis: cohort study using linked data. Br J Cancer 111: 1490-1499.

Singh H, Daci K, Petersen LA, Collins C, Petersen NJ, Shethia A, El-Serag HB (2009) Missed opportunities to initiate endoscopic evaluation for colorectal cancer diagnosis. Am J Gastroenterol 104: 2543-2554

Tsang C, Bottle A, Majeed A, Aylin P (2013) Cancer diagnosed by emergency admission in England: an observational study using the general practice research database. BMC Health Serv Res 13: 308.

von Wagner C, Baio G, Raine R, Snowball J, Morris S, Atkin W, Obichere A, Handley G, Logan RF, Rainbow S, Smith S, Halloran S, Wardle J (2011) Inequalities in participation in an organized national colorectal cancer screening programme: results from the first 2.6 million invitations in England. Int J Epidemiol 40: 712-718.

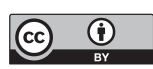

This work is licensed under the Creative Commons Attribution 4.0 International License. To view a copy of this license, visit http://creativecommons.org/licenses/by/4.0/

Supplementary Information accompanies this paper on British Journal of Cancer website (http://www.nature.com/bjc) 